\title{
UNAS NOTAS CRITICAS AL TEXTO DE PACIANO DE BARCELONA
}

Some emendations, restitutions and critical remarks to the text of Paraenesis ad Paenitentiam which was originally entitled De Paenitentibus.

I. Título DE LA OBRA LLAMAdA COMÚNMENTE "Paraenesis ad Paenitentiam".

Desde la Editio Princeps el opúsculo de Paciano relativo a los penitentes suele conocerse bajo el título Paraenesis siue Exhortatorius Libellus ad Paenitentiam, que impuesto por Jean du Tillet, fue adoptado por la inmensa mayoría de las ediciones ${ }^{1}$. Galesino, no obstante, introdujo una modificación y lo llamó De Paenitentia et Confessione Libellus. $\mathrm{K}$. Barth en la edición ${ }^{2}$ sigue a du Tillet parcialmente con Paraeneticus ad Poenitentiam que en sus Adversaria ${ }^{3}$ abrevia simplemente en $\mathrm{Pa}$ raeneticus. Aguirre ${ }^{4}$ es el primero en aludir a la tradición manuscrita con una nota marginal donde dice: De paenitentibus Vat. R. Kauer ${ }^{5}$

1 Una relación de las adiciones de Paciano puede verse en L. Rubio Fernándeż, San Paciano. Obras, Universidad de Barcelona, 1958, pp. 40-43 y en T. G. Schoenemann, Bibliotheca Histórico-Literaria Patrum Latinorum, Lipsiae, I792, I, pp. 357-361.

2 S. Paciani Episcopi Barcinonensis, Paraeneticus ad Poenitentiam cum Claudiani Mamerti libris III de statu animae..., Zwickau, 1655, pp. 934-952.

3 Adiersariorum Commentariorum Libri L.X, Frankfutt, 1648, lib. XLIII, cap. XV, col. 1956 .

- Collectio Maxima Conciliorum Omnium Hispaniae, Roma, 1753, I, p. 336, n. 4 .

s Studien zu Pacianus, en Jahresber. des $k$. k. Staatsgymnasiums im XIII Bez., Wien, 1902, p. I, n. 1 . 
enumera los escritos de Paciano según el orden de $\mathrm{R}^{1}$ y pone en primer lugar el De paenitentibus. J. W. Ph. Borleffs ${ }^{2}$ observa con razón que Paraenesis ad Paenitentiam carece de fundamento en los manuscritos, porque en estos reza $D e$ paenitentibus. También se repite en $\mathrm{L}$ y $\mathrm{V}$, testigos de la Collectio ex dictis XII Patrum ${ }^{3}$.

De hecho Paciano, cuando en su opúsculo se refiere al contenido, nunca da a entender que se trate de una exhortación o Paraenesis, antes bien dice: de paenitentium curatione ${ }^{4}$, hunc ipsum de paenitentiae institutione sermonem ${ }^{5}$. El título de los mss. resulta mucho más acorde con el De Paenitentia de otros opúsculos sobre el mismo tema escritos por autores como Tertuliano y S. Ambrosio entre otros. Además de Tertuliano, influye grandemente en nuestro escrito Cipriano con su De Lapsis. Esta circunstancia explica mejor que Paciano no copiara exactamente el título de la obra de Tertuliano, sino que usara un sinónimo de De Lapsis como es De Paenitentibus ${ }^{6}$. Así se llamará en nuestras sucesivas investigaciones.

El título es, pues, con toda certeza De paenitentibus.

\section{PAEN. II I}

\section{Transcripción del pasaje per cola et commata segin $R^{7}$}

Atque ideo adblandam huius mei operis ac sollicitam diligentiam.

et secundum domini uoluntatem a me ipso fratre uestro ac sacerdote susceptam,

amorem potius credite quam rigorem.

Sequendo amabiliter.

non pertinaciter repugnando.

1 La sigla R corresponde al ms. Reginensis 33r. Cf. Angel Anglada, "La tradición manuscrita de Paciano de Barcelona", Emerita 35,1967, pp. $137 \mathrm{~s}$.

"Zwei nete Schriften Pacians?", Mn. 7, 1939, p. 180, n. I.

3 Angel Anglada, op. cit., p. $138 \mathrm{~s}$.

- Paen. I I. Las referencias remiten siempre a la edición de I. Kubio Fernández citada en la primera nota de este artículo.

5 Paen. II 2.

- Sobre la igualdad de significado entre lapsus y paenitens véase $\mathrm{H}$. Koch, Cyprianische Untersuchungen, Bonn, 1920, p. $380 \mathrm{~s}$.

7 Acerca de la escritura per cola et commata en $\mathrm{R}$ puede consultarse $\mathrm{R}$. Kauer, $o p$. cit., p. 2, n. 2 y Angel Anglada, "La puntuación del ms. Reginensis 33 I en el texto de Paciano de Barcelona", Vetera Christianorum I2, I975, pp. 269-316. 


\section{El testimonio de los mss.}

La transcripción anterior reproduce exactamente el texto de $\mathbf{R}$. $\mathrm{G}$ lo copió con toda fidelidad. Fue $\mathrm{P}$ el primero en escribir por separado ad blandam. Los mss. de la Collectio ex dictis XII Patrum no extractan este texto.

\section{El texto de las ediciones}

a) Editio princeps

Jean du Tillet repite el texto de $\mathrm{R}$ sin variar las pausas, pero no entiende adblandam $\mathrm{y}$, como si ad fuera una preposición, corta ad y blandam. La misma división publica Galesino, quien añade algunas comas por su cuenta.

\section{b) Otras ediciones}

La impresión por separado de ad y blandam ha motivado en buena parte la supresión de ad en la mayoría de las ediciones, las cuales se limitan a escribir simplemente blandam. Tal es el caso, por ejemplo, en las de Maguerin de la Bigne ${ }^{1}$, Barth, Gallandius, Noguera, Migne, Flórez. En el texto de Aguirre se reproduce blandam, pero en el margen remite al Reginensis, que, por cierto, no leyó muy bien, pues la nota dice así: $A d$ bla. Vat. Como puede observarse atribuye erróneamente a $\mathrm{R}$ la separación ad blandam.

c) Ph. H. Peyrot y L. Rubio

Peyrot también sintió ad como preposición, y la puso entre corchetes, no sin hacer esta indicación en el aparato crítico: ad $P R$ Tilius. deleui cum edd. No es esta la primera vez que Peyrot se equivoca en la lectura de $\mathrm{R}^{2}$. Intenta justificar la supresión de ad trayendo a colación uno de los dos pasajes en que Paciano usa el adjetivo blandus: tunc amares (scil. me) si... operi meo blandus accederes ${ }^{3}$. El otro pasaje

1 Paris, I575, I, col. 324 E; Paris, I589, III, col. 451 D; Paris, 16r8, IV, p. 244 E; Lyon, 1677, IV, p. 314 D.

- Véase principalmente L. Rubio Fernández, "El texto de San Paciano", Emerita 25, 1957, pp. 330-332.

- Ep. II 7, 2. 
es: filio reuertenti... pater blandus ocurrit ${ }^{1}$. Estas referencias confirman, desde luego, el uso de blandus en Paciano, pero no proyectan luz alguna sobre el problema de crítica textual que nos ocupa. Rubio se limita prudentemente a encerrar entre corchetes ad e indicar en el aparato crítico su supresión en las ediciones.

\section{Consideración estilistica}

Entre los dos pasajes que se acaban de citar y el texto que estudiamos media una diferencia importante. Aparece blandus en la correspondencia con Simproniano, persona culta, que en la primera carta del obispo barcelonés supo descubrir un verso de la Eneida y en su admiración apasionada por Virgilio llegó incluso a reprochar a Paciano una inversión en el orden de las palabras ${ }^{2}$. Inversión que, por cierto, convertía el ritmo de poesía en ritmo de prosa ${ }^{3}$. En la respuesta a un uir eloquens, Paciano replicó con un lenguaje más elevado que el dẹ la predicación al pueblo sencillo.

\section{Consideración paleográfica}

Entendido como preposición, ad viene ofreciendo dificultad desde Jean du Tillet, que editó ad blandam, acrecentada ésta por P y Peyrot.

La supresión de ad no parece fundamentarse en unos cimientos paleográficos muy firmes. De hecho, $\mathrm{R}$ no presenta en el texto de $\mathrm{Pa}$ ciano errores de copista constitutivos de una falta que añada una preposición contraria a la sintaxis de nuestro escritor. Algunos errores de esta fndole son introducidos por la mano de Florus de Lyon ${ }^{4}$. Si bien $a d$ se halla en una palabra abundante en letras de trazos similares, no deja de ser aventurado suponer en el escritor un error de cálculo que le llevase a agregar cuatro palos de más. Paleográficamente, la pre-

\footnotetext{
1 Ep. I $5,8$.

- Cf. L. Rubio Fernándes, San Paciano. Obras, Barcelona, 1958, pp. 2 I s. y 25 .

R. Kauer, op. cit., p. 8. Esta importante circunstancia ha pasado desapercibida a S. Costanıa, "La polemica di Paciano e Simproniano sull'uso di citare i poeti classicin, Vetera Christianorum I5, 1978, pp. 45-50, particularmente p. 48 .

- Sobre las correcciones de Florus de Lyon puede verse Angel Anglada; «La tradición manuscrita de Paciano de Barcelonas, EMmerita 35, 1967, pp. I40I54.
} 
sencia de ad no se explica por una falta de amanuense y, por tanto, su supresión sin más carecería de base.

\section{Consideración etimológica}

Ante el hecho de la lectura adblandam y no ad blandam en el ms. de la Reina de Suecia, no cabe pensar en una preposición ad infringiendo las normas más elementales de la sintaxis latina, sino en un adjetivo adblandus, $-a,-u m^{1}$. Nada obsta que no se halle en los léxicos latinos ni siquiera en el $T h L L$, ni tampoco en las. Addenda de su Zettelarchiv o en el Glossarium de Du Cange o en los diccionarios etimológicos de las lenguas románicas ${ }^{2}$. Basta el testimonio de la tradición manuscrita, aunque ésta atestigue un apaxlegómenon ${ }^{3}$.

No resulta absurda en modo alguno la lectura adblandam si se recuerda la existencia en castellano del verbo ablandar. Los diccionarios etimológicos de la lengua castellana ${ }^{4}$ suelen reducir la etimología de 'ablandar' a la composición de $a$ y el latín blandiri o de $a$ y blando y éste del latín blandus. Si el catalán ablanir deriva del latín adblandiri no cabe decir lo mismo sin más del castellano ablandar. Mucho más lógico parece buscar el origen en el latín vulgar adblandare, no conservado, pasando por la correspondiente asimilación de $d$ a $b$ dando abblandare para simplificarse luego la geminada en ablandare, que remonta al adjetivo adblandam, atestiguado sólo por Paciano de Barcelona en un escrito dirigido al pueblo.

1 Un caso inverso se encuentra en Horacio, Serm. II I1, 3. Cf. P. Lejay, Lexicographie Latine. I Ab normis non abnormis, en Mélanges Boissier, París, 1903, p. $345 \mathrm{s.}$

F. Diez, Etymologisches Worterbuch der Romanischen Sprachen. Bonn, 1877; W. Meyer-Lübke, Romanisches Etymologisches Wörterbuch, Heidelberg, 193.5.

so era insólito que en los compuestos de ad el copista sintiera el preverbio como preposición y lo escribiera separado. Así el subjuntivo adlaborem es atestiguado por los mss. M P X de Tertuliano De Patientia con el preverbio separado ad laborem, según puede observarse en el aparato crítico de la edición de $\mathrm{J}$. W. Ph. Borleffs, Tertulliani De Patientia XIII, I (CCh I, p. 313 s.).

También, por ejemplo, la palabra demorator existe únicamente en Marciano Capella con la sola base de la tradición manuscrita. Cf. Jean Preaux, Le culte des muses chez Martianus Capella, en Mélanges de Philosophie, de Littérature et d'Histoire ancienne offerts à Pierre Boyancé, Roma, 1974, pp. 593-597.

- El Diccionario Crítico Etimológico de J. Corominas no estudia ablandar. 
Conclusión

A tenor de las consideraciones precedentes, se impone restablecer el texto según la lectura transmitida por $\mathrm{R}$, leyendo adblandam y no [ad] blandam como Peyrot, y mucho menos ad blandam como P y Jean du Tillet. Con esta fidelidad a la tradición manuscrita se recupera un adjetivo del latín vulgar existente sólo en la península ibérica.

III. PAEN. II 2

I. Transcripción del pasaje per cola et commata según $R$

Praeterea nullus existimet.

hunc ipsum de penitentiae institutione sermonem.

solis tantummodo penitentibus ordinatum.

Ne propter hoc quisquis extra hunc gradum positus est.

ea quaecumque dicentur uelut in alios destinato fastidiat.

Cum in hanc quasi fibulam.

totius ecclesiae disciplina notetur.

Quando et cathecuminis ne in hoc transeant.

et fidelibus ne in hoc redent prouidendum sit.

Ipsis uero paenitentibus.

ut celeriter ad fructum huius operis perueniant laborandum.

2. Testimonio de los mss.

Las líneas anteriores reproducen el texto tal como se presenta en $\mathrm{R}$. Florus que no aprovechó este pasaje para su Collectio ex dictis $\mathrm{XII} \mathrm{Pa}$ trum y, por tanto, no se encuentra en L V. El ms. G copia el texto de R con la enmienda del propio copista del Regionensis 33r, como es destinata por destinato y con la corrección floriana redeant por redent. Las variantes de $G$ se reducen a la omisión o añadidura de algún signo de pausa. El ms. P sigue el texto de $\mathrm{G}$ no sin variar ligeramente también la puntuación.

\section{Ediciones}

a) Editio princeps

Jean du Tillet imprime fielmente el texto de $\mathrm{R}$ con las dos enmiendas ya indicadas, pero en el margen interpreta notetur como coarctetur. 
Esta nota marginal, alias coarctetur, ha sido un escollo para algunos editores posteriores. Así Ga.esino fue el primero en escribir nodetur en vez de notetur, modificación que luego muchos han seguido. M. de la Bigne en sus cuatro ediciones, Barth y Galland, en cambio, conservan notetur, no sin reproducir a veces la interpretación Tiliana en una nota marginal o a pie de página.

\section{b) K. Barth}

En sus Adversaria ${ }^{1}$ Barth escribe esta interesante observación respecto de fibula según aparece en el paso que comentamos: «Fibula pro Enchiridio aliquo aut tabella memoriali sive Breviculo, ut vocant, aut laterculo, in quo aliquid memoriae gratia notatur, posita est his verbis. 'Praeterea nullus existimet... disciplina notetur' ${ }^{2}$. Servat Germanismus hanc vocem. Fibulam enim dicimus ex qua pueri primum elementa litterarum agnoscere et pronunciare discunt. Sic Tertullianus fibulam vocat scultellum sive rationale illud quod portabat Aaron lib. de Idololatria cap. XIX, 2 Virgam portavit Moyses, fibulam et Aaron, congitur loro et Ioannes, agmen agit et Iesus Nave». Barth interpreta, pues, fibulam de Paciano como el alemán Fibel y en confirmación de este sentido aduce un texto de Tertuliano.

\section{c) J. Fr. Gronov}

Gronov tilda de absurda la interpretación de Barth arguyendo que el alemán Fibel es una corrupción del griego $\beta$ íp Bibel, es decir, la Sagrada Escritura. Entiende Fibula en Paciano como hebilla, a la que corresponde con toda propiedad nodare. Con una cierta reserva y no mucho acierto insinúa sustituir el acusativo por el ablativo in hac quasi fibula. Escribe así el erudito holandés ": "Pro notetu*, alii legunt coarctetur. Alii observant Germanismum vocem fibulae sezvare pro libello seu tabellis, in quibus quid notetur vel scribatur: ita enim dici ex qua pueri elementa literarum agnoscere et pronuntiare discant. Quis autem potest dubitare, Germanorum fibel esse corruptum, non ex Latino fibula, sed ex Graecorum $\beta$ ip $\lambda_{\text {os }} \hbar \beta_{1} \beta \lambda$ iou ut et bibel pro scriptura sacra? In Paciano legimus: cum in hanc quasi fibulam

1 Lib. XLIII, cap. XV, col. $1957 \mathrm{~s}$.

2 Corresponde al texto de Paciano que se estudia.

3 Johannis Frederici Gronovii, Observatorum in Scriptoribus Ecclesiasticis Monobiblos, Draventiae, 1651, p. $193 \mathrm{~s}$. 
totius Ecclesiae disciplina nodetur. Vel, in hac fibula. Ipsam poenitentiam sic vocat, quod omnem contineat Ecclesiae disciplinam; cuius expedit $\&$ catechuminis $\&$ fidelibus $\&$ poenitentibus recordatione comprimi, retineri \& morderi; perinde ut fibula nodat $\&$ mordet $\&$ continet vestem. Nodare verbum proprium de fibula. Sic Claudiano, tereti nodantur jaspide vestes. Idem verbum restituendum Adhelmo cap. IX. Iugalitas, quae ad procreandam posteritatis subolem \& liberorum procreandorum gratia licitis connubij nexibus credatur. Legendum, nodatur. Sic enim infra de Chrysantho: hortantur patrem, ut filius blandis connubij nexibus nodaretur. De Davide: antequam copula matrimonij et connubij nexu innodaretur. De Samsone: deceptus illecebrosis lenocinij nexibus nodareturn.

\section{d) Noguera}

Noguera ${ }^{1}$ entiende fibula también como la hebilla con que los antiguos se ceñfan el vestido y ve confirmada su interpretación en la nota marginal de Tilius, por lo cual prefiere la lectura nodetur de Galesino al notetur del Reginensis, de la editio princeps y de Aguirre. Dice así: "Nodetur. Ita exhibet editio Romana; Paris. vero, Matrit. Aguirr. quin et Cod. Vatican. notetur habent; at Paris. ad marginem posuit alias coarctetur. Placuit tamen Rom. praeferre lectionem, nam fibula vestimenta subligabant veteres, eaque subnectebant; quod verbo nodetur satis superque exprimitur.»

\section{e). J. van der Vliet y Ph. H. Peyrot}

J. van der Vliet ${ }^{2}$, más bien dentro de la línea de Gronov, precisó un poco más la interpretación de hebilla. Vio igualmente en fibula un sentido metafórico que responde a una supuesta comparación de la disciplina penitencial con el vestido cuyos pliegues la hebilla mantendria adaptados al cuerpo. Sobre la base de esta pretendida metáfora adopta la enmienda nodetur de Galesino. Dice asi: "Illa (la penitencial) disciplina comparatur cum vestimento, cuius sinus una fibula continetur. Scribo autem: cum in hanc quasi fibulam totius ecclesiae disciplina nodetur.» En confirmación aduce el único verso donde Virgilio $^{3}$ usa nodari en un contexto donde aparece la palabra fibula, aunque como

D. Paciani Episcopi Opera quae extant, Valencia, 1780, p. 220 C.

-Ad Sancti Paciani Opuscula», Mn. 23, 1895, p. 196.

Aen. IV, p. $138 \mathrm{~s}$. 
complemento de subnectit: ...crines nodantur in aurum / aurea purpuream subnectit fibula uestem. Peyrot, naturalmente, repite nodetur, que atribuye sin más a van der Vliet, pues anota en el aparato crítico: «nodetur pro notetur v. d. Vliet» y en la nota explicativa dice: (in fibulam... nodetur emendatio egregia uiri cl. v. d. Vliet» y añade: "nodari in Pacianus inuenerat apud Vergilium, quem in deliciis habebat eumque 'a paruulo didicerat'" ${ }^{1}$.

\section{Fibula como tecnicismo retórico}

En latín fibula no tiene solamente el sentido de 'hebilla' para abrochar o sujetar el vestido. Es también un término técnico de la oratoria y del lenguaje de los astrónomos.

En su carta dirigida al emperador canta Frontón ${ }^{2}$ las glorias del humo, del polvo y del descuido, y en los prolegómenos sobre la manera de escribir usa la palabra fibula en un sentido técnico retórico de conclusión añadida elegantemente a un texto breve: ut nouissimos in epigrammatis versus habere oportet aliquid luminis, sententia claui uel fibula terminanda est ${ }^{3}$.

También Tertuliano ${ }^{4}$ conoce el significado gramatical de fibula, cuando dice: "autem» ipsum uelut fibula. Juega, además, con su doble significado introduciendo el verbo alligat. Dice asi el presbítero cartaginés: Nam et "autem» ipsum uelut fibula coniunctiuae particulae ad conexum narrationi adpositum est: "terra autem». Hoc enim uerbo reuertitur ad eam de qua supra dixerat, et alligat sensum. Blaise ${ }^{5}$ da esta referencia para el sentido de "particule conjonctive".

E1 mismo juego de palabras combinàndo el doble sentido de fibula como texto y como hebilla aparece en otro pasaje del mismo escritor, donde después de citar dos versículos de la primera carta a los corintios, alude a ellos llamándolos fibula, que resulta ser, además, complemento de relaxasse. E1 pasaje reza así ${ }^{6}$ : De quibus autem scripsistis bonum est

1 Por la forma de expresarse v. d. Vliet se comprende que Peyrot le atribuya la paternidad de la lectura nodetur de Galesino.

- Laudes fumi et pulueris 2. Cf. M. P. J. van den Hout, M. Cornelii Frontonis Epistulae, Leiden, 1954, I, p. 201.

3 Véase ThLL VI, col. 645 , fibula 2 b.

- Hermog 26, 3 (CCh I, p. 4r9).

s Dictionnaire Latin-Franiais des auteurs chrôtiens, Turnhout, 1954, p. 350 , fibula.

- Pud. XVI, r3 s. (CCh II, p. 1313). 
homini mulierem non contingere; sed propter fornicationem unusquisque uxorem suam habeat; uir uxori et uxor uiro debitum reddat. Huius boni fibulam quis illum nesciat inuitum relaxasse, ut fornicationi obuiam esset?

La interpretación de Blaise entendiendo aquí fibulam relaxasse como el opuesto a fibulam imponere, supone negar un juego de palabras, precisamente cuando fibulam se refiere a un texto bíblico. Blaise por otra parte, no menciona en absoluto el sentido retórico de fibula. De modo parecido Ennodio ${ }^{1}$ combinará la mordacidad de las palabras de la fibula en sentido retórico, con la tenaza de la hebilla en: subiunctam quaestionem rhetorica fibula momordistis allegando: "Testis est Romana ciuitas, si omnes episcopi senes et debiles conuenerunt».

\section{Fibula en el Computo Pascual Cartaginés del año $455^{2}$}

a) Transcripción del pasaje según $\mathrm{Br}$. Krusch

His quoque supradictis columnis aliae septem iunguntur, in quibus kalendarum ianuariarum luna et pascae dominicorum dies adnotantur. Tricesimarius etenim lunae numerus per septenarium numerum ebdomadis diuisus, spatias numero ducenta decem disterminat. Ut autem uniuscuiusque paschae diem inuenias, lunam et feriam, quae fuerit kalendis ianuariis obseruabis, ut lunae cursum tenens, in eadem columna diem inuenies paschae, quod ad feriam suprascriptam pertinere uidetur. Hoc idem et in fibulis triginta sequentibus adprobatur, in quibus kalendarum martiarum lunam et feriam per septenarium numerum utrarumque in lunae dierum triginta, ducenta decem spatia inuenies adnotata, nuntiantes certissime fixum uniuscuiusque anni dominicum pasche. Nam et harum fibularum numerum tres aliae ut pediseque columnae secuntur, quae in se embolismorum titulos et lunares annos, lineis tensis ex kalendis ianuaris, quota fuerit, curiosis et bene sullicitis planius monstrare coguntur, ut horum circulorum ueritas in omnibus suis ornata monilibus ostendatur ${ }^{3}$.

\section{b) Comentario}

E1 sentido de fibula en este pasaje es correlativo de adnotata, pues el correlativo de in quibus es fibulis. El significado de adnotare incluye

1 Opusc. 2, 12; PL LXIII, col. 185.

- La época del Cómputo Pascual Cartaginés está tomada de Br. Krusch, Studien sur christlich-mittelalterlichen Chronologie. Der 84 iähriger Ostercyclus und seine Quellen, Leipzig, 1880, pp. 138-150.

- Cf. Krusch, op. cit., p. 285 . 
el concepto de indicación breve. Por otra parte, estas fibulae son como las columnas que permiten reconocer fácilmente los años del saltus lunae en el cómputo pascual. Hay anotación lo mismo en las columnae que en las fibulae, como se deduce de columnis aliae septem iunguntur in quibus... adnotantur. Que las anotaciones eran breves lo evidencia también la frase quae in se embolismorum titulos et lunares annos lineis tensis... monstrare coguntur. La circunstancia de escribir en rojo los días de la semana de los años de salto lunar para que destacaran, contribuye también a poner de manifiesto el carácter esquemático de las indicaciones. De estas observaciones se desprende que para el computista cartaginés fibula no es, desde luego, una hebilla que abrocha el vestido, sino un escrito breve a modo de sinopsis y resumen.

El paralelismo de significado de fibula entre el computista cartaginés, de mediados del siglo $\mathrm{v}, \mathrm{y}$ Paciano resulta más relevante por coincidir incluso los verbos adnotare: notetur.

\section{Etimología del alemán Fibel}

Los diccionarios etimológicos alemanes ${ }^{1}$ derivan la palabra Fibel de Bibel por la razón de que la cartilla escolar donde los niños aprenden a leer contiene muchas historias tomadas de la Biblia. Así Fibel tomaría su origen de una pronunciación infantil que deformaría la primera $b$ de Bibel en $f$ dando. Fibel.

De hecho, la palabra alemana Fibel aparece atestiguada por primera vez a principios del siglo $\mathrm{xv}$ y más concretamente en un plan de enseñanza de las clarisas de Kibnitz en Rostock ${ }^{2}$. Un vocabulario de Indersdorf (Munich) del año I4I9 traduce "Abecedarium, ein ABC oder ein Fibel\%. Es particularmente interesante el significado de 'sermón de reprimenda' (Strafpredigt) o amonestación basada en un texto bíblico. Es este un sentido muy afín al de fibula en el De Paenitentibus, en donde Paciano amonesta con textos bíblicos a sus fieles que no cumplen debidamente la penitencia.

$\mathrm{K}$. Barth abunda en esta interpretación cuando estima que el alemán Fibel es la misma palabra latina fibula usada ya por Tertuliano en el sentido de cartilla. El significado de 'cartilla' del latín fibula des-

1 Cf., por ejemplo, F. Kluge, Etymologisches Wörterbuch der deutschen Sprache, Berlín, 1967, p. 196; Der grosse Duden 7. Herkunftswörterbuch, Mannheim, 1963. p. 166; y sobre todo, Alfred Edbe, Trübners deutsches Wörterbuch. In Auftrag der Arbeitsgemeinschaft für deutsche Wortforschung, Berlin, 1940, p. 340 s.

2 MG Paed. 38, 47. 
virtúa, por lo menos, la etimología tradicional del alemán Fibel y hace pensar en su origen latino junto con Barth.

Con la etimología Fibula para Fibel concuerda materialmente D. Sanders ${ }^{1}$ cuando observa que algunos derivan Fibel de Fibula en el sentido de la grapa usada en la encuadernación de los libros. Así cita la frase Seine arme Fibulisten de Lutero 8, 237a. M. Heyne en su Deutsches Wörterbuch atribuye a glosas antiguas la etimología Bibel, dato que quita posibilidad a ésta.

\section{Consideraciones estilisticas}

Si para fibula se admite el sentido de texto breve, notetur no ofrece la menor dificultad. La frase 'anotar en un texto' une conceptos correlativos y notetur es tan apropiado para un texto, como 'abrochar' para una hebilla.

Podría objetarse que disciplina nodetur constituye una cláusula $1 \gamma$ frente al final de hexámetro que supone disciplina notetur. A esta hipotética dificultad cabría responder que si bien el porcentaje de la cláusula $1 \gamma$ es en Paciano incomparablemente superior a los demás ritmos de distinctio plena, el grado de pausa de notetur apenas si llega a ser de distinctio media, y por cierto, menor al de prouidendum sit, que a su vez es inferior a la de laborandum. Cabe recordar que en estas pausas la ley de la cláusula no depende únicamente del grado de distinctio y Paciano pudo haber evitado un ritmo propio de final, más normal en distinctio plena ${ }^{2}$.

\section{Conclusion}

La lectura notetur de $\mathrm{R}$ no contraviene ni al sentido ni al contexto léxico tradicional de fibula. Se impone, pues, su conservación y debe leerse: cum in hanc quasi fibulam totius ecclesiae disciplina notetur. Su traducción es: "ya que en este por así decir prontuario se anota la enseñanza de la Iglesia entera».

1 Wörterbuch der deutschen Sprache, Mit Belegen von Luther bis auf die Gegenwart, Leipzig, I860, I, p. 44I; M. Heyne, Deutsches Wörterbuch, Leip $\measuredangle i g$, I905, I, col. $908 \mathrm{~s}$.

El autor de estas páginas espera poder publicar ya pronto un estudio sobre este tema. 
IV. PAFN. V 2-3

I. Transcripcion del pasaje per cola et commata según $R$

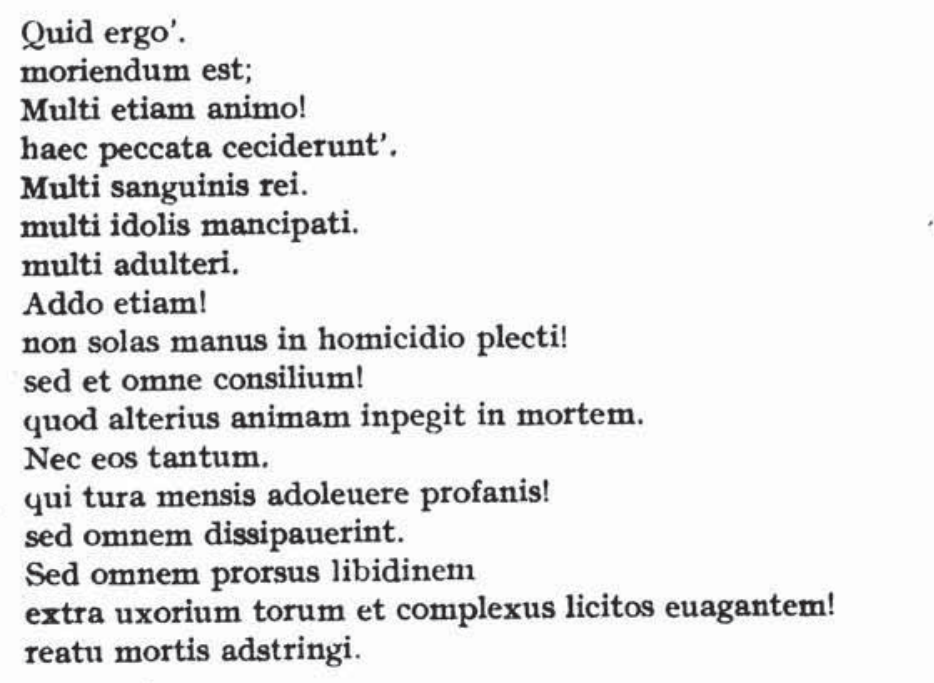

\section{Testimonio de los mss.}

El rexto de $\mathrm{R}$ está reproducido exactamente en las líneas precedentes. Florus de Lyon corrigió haec peccata en IN haec peccata sobreañadiendo IN entre animo y haec. Esta enmienda es recogida por la tradición manuscrita entera y por todas las ediciones. En el margen de $\mathbf{R}$ puso Florus una $r$ de require concerniente al miembro viciado sed omnem dissipauerint. Los mss. L V extractan en el pasaje correspondiente este miembro sin indicación marginal alguna. Los mss. G P copian la $r$ floriana en el margen.

\section{Ediciones}

\section{a) Editio princeps}

Jean du Tillet prefirió omitir sin más el ininteligible sed omnem dissipauerint, con lo cual quedó eliminado también de todas las ediciones posteriores a las que sirvió de modelo y vulneró así una vez más el texto en un punto importante, como es la omisión de un miembro 
en un catálogo de pecados. También falta en Noguera, aunque éste no dependió exclusivamente de la edición tiliana ${ }^{1}$, sin que se lea nota alguna al respecto.

\section{b) Otras ediciones}

Nuestro colon sed omnem dissipauerint reaparece en las ediciones verdaderamente críticas y que recurren directamente al testimonio de los mss., como son la de $\mathrm{Ph}$. H. Peyrot, pese a tantas inexactitudes y enmiendas arbitrarias ${ }^{2}$ y sobre todo la de L. Rubio Fernández.

Para J. van der Vliet ${ }^{3}$ entre profanis y sed omnem prorsus libidinem mediaban, evidentemente, dos miembros que se perdieron. Argumenta que a la mención de los idólatras debía seguir la de unos adoradores de otros dioses paganos. De hecho no parece muy lógico distinguir a los idólatras de otros adoradores de dioses paganos, cuando la enumeración es de carácter tan general. El latinista de Leiden escribe así: "Facile uisu est, inter profanis et sed omnem duo membra periisse, nam iis qui idolis sacrificent, iungendi erant illi, qui alia quacumque ratione deorum paganorum cultui uel faueant uel seruiant, de quibus agit Tertullianus in libello de Idololatria, illis uero qui castimonii legibus non obtemperent, ut pessimum genus praemittendi erant adulteri».

$\mathrm{Ph}$. H. Peyrot abunda en esta observación de su maestro e introduce a los adúlteros delante de sed omnem prorsus con el texto modificado así: sed omnem dissipauerint... nec modo adulteros sed omnem prorsus...

L. Rubio Fernández ${ }^{4}$ estudia la presente corrupción y piensa que es muy breve y alcanza a un solo miembro. Este resultado preside en buena parte nuestras líneas.

\section{c) A. Gruber}

A. Gruber en sus Studien zu Pacianus von Barcelona ${ }^{5}$ considera

\footnotetext{
1 D. Paciani Episcopi Barcinonensis Opera quae extant, Valencia, I780, p LXX s.

2 Véanse las recensiones de C. Weymann, en $B P h W 16,1896$, cols. 10571061 y 1104-1108; G. Pfeilschifter, en WklPh 13, 1896, cols. 1112-1116 y Wilde, en Museum, 1896, IX. Son favorables sólo las brevisimas reseñas en $A L L$ Io, 1898, p. 307 y la alusión de la nota necrológica en honor de J. v. d. Vliet, en Jahrbuch für Altertumswissenschaft 24, 1903, p. 98. Véase sobre todo L. Rubio Fernández, El Texto de San Paciano citado en p. 13, n. 2 de este artículo.

3 Ad Sancti Paciani Opusculaw, Mn. 23, 1895, p. 196.

- El Texto de San Pacianon, EMERITa 25, 1957, p. 363.

- P. $35 \mathrm{~s}$.
} 
insuperable la dificultad de este pasaje, que estima completamente corrupto. Elogia la agudeza y acierto de van der Vliet por haber descubierto tres pensamientos, plasmados cada uno de ellos en dos miembros contrapuestos: $I$. non solas manus - sed et omne consilium; II. nec eos tantum qui thura mensis... profanis, como segundo miembro está el sed omnem dissipauerint evidentemente corrupto; III. en el tercer pensamiento Peyrot suplió el primer miembro que falta con la excelente corrección, nec modo adulteros'. Segundo miembro: sed omnem prorsus libidinem... euagantem. Sin pretender llegar a una restitución definitiva, Gruber intenta proyectar un haz de luz sobre el miembro sed omnem dissipauerint, en el que no entraron van der Vliet ni Peyrot. Así recurre a las fuentes principales del escritor barcelonés, como son Tertuliano y Cipriano. Para ello trae a colación un pasaje de Tertuliano ${ }^{1}$ que dice: sed omnem afflatum eius (idololatriae) ${ }^{2}$ uice pestis etiam de longinquo deuitemus. A tenor de estas palabras Gruber propone como posible la conjetura: nec eos tantum qui... sed omnes qui non etiam afflatum idolatriae vice pestis de longinquo deuitauerint. E1 texto de Cipriano aducido por Gruber es: qui quamuis nullo sacrificii aut libelli facinore constricti, quoniam tamen de hoc uel cogitauerunt, hoc ipsum apud sacerdotes Dei dolenter et simpliciter confitentes ${ }^{3}$. A la vista de estas frases piensa Gruber en leer: nec eos tantum qui... sed omnes, qui de hoc uel cogitauerint. Para justificar mejor su hipótesis añade, no sin cierta reserva, que paleográficamente ni deuitauerint ni cogitauerint quedan lejos de dissipauerint.

A nuestro entender este pasaje contiene una enumeración de pecados: E1 homicidio non solas manus in homicidio plecti, la idolatria qui thura mensis adoleuere profanis, otro irreconocible a primera vista bajo el sed omnem dissipauerint. Finalmente, el adulterio, sed omnem prorsus libidinem, extra uxorium thorum et complexus licitos euagantem. El pecado de adulterio es considerado por los antiguos cristianos como un pecado de idolatría. Asi dice Tertuliano ${ }^{4}$ : qui falsis deis seruit, sine dubio adulter est ueritatis, quia omne falsum adulterium est. Sic et stupro mergitur. Quis enim immundis spiritibus cooperatur non conspurcatus et constupratus incedit? Atque adeo scripturae sanctae stupri uocabulo utuntur in idololatriae exprobratione. Por otra parte, también el idólatra es

1 La referencia dada por Gruber de idolatr. c. 13 corresponde a c. 12, 3 ( $C C h$, p. III2).

3 Gruber escribe idolatriae.

3 Laps. 28 (CCh III, p. 236).

- Idol. I 2 (CCh. II, p. IOOI). 
homicida ${ }^{1}$. Los pecados mencionados en nuestro pasaje son, pues, pecados que participan grandemente de la idolatria.

Gruber para sostener que en cada pensamiento hay dos miembros contrapuestos entre si, se ve obligado a recurrir al texto de Peyrot y darlo por bueno. Quizás sería más razonable pensar en la figura de la gradatio, pues el primer pensamiento está formado por dos miembros que se contraponen mediante sed. En el segundo pensamiento al acto externo de quemar incienso se contraponen mediante dos sed el corrupto sed omnem dissipauerint y el adulterio, otra forma de idolatría. E1 miembro relativo al adulterio no requiere, pues, otro miembro propio al cual se oponga, como supone la conjetura de Peyrot, antes bien junto con el inmediato anterior se contrapone a la idolatría material expresada en nec eos tantum qui thura mensis adoleuere profanis. Si Gruber recurre a un supuesto parecido paleográfico entre dewitauerint o cogitauerint es porque ve la corrupción en dissipauerint. Parte, por tanto, como los editores anteriores, de que sed omnem ha sido transmitido incólume y sin error. La expresión sed omnem es quizás demasiado común y a la vez poco consistente para que se desplace la corrupción al dissipauerint, menos conocido probablemente de los copistas medievales.

\section{d) L. Wohleb}

L. Wohleb ${ }^{2}$ corrigió la corrupción a base del ad Fortunatum $^{3}$ de Cipriano en esta forma: sed (et) omnes (qui sacrificare et seruire idolis suaserint). Esta enmienda carece igualmente de fundamento paleográfico y el miembro obtenido resulta demasiado largo para las proporciones de los demás dentro de la armonía del contexto.

\section{Isocolia}

$\mathrm{Si}$ se compara la longitud de los miembros que empiezan por sed a la luz de la puntuación conservada por el Reginensis ${ }^{4}$ se observa

1 Tert., Idolol. I, I-2 (CCh II, p. IroI). C. Weymann reunió algunas expresiones paralelas a qui thura mensis adoleuere profanis en "Miszellen: Similia zu Vergils Hirtengedichten VIv, WS $46,1927-28$, p. ror.

- "Bischof Pacianus von Barcelona und sein Gegner der Novatianer Sympronianus», Gesammelte Aufsütze zur Kulturgeschichte Spaniens 2, 1930, p. 29, n. 13.

8 Fort. 5 (CCh III, pp. r9I-r93).

- Cf. Angel Anglada, "La puntuacion del ms. Reginensis 331 en el texto de Paciano*, Vetera Christianorum 12, 1975, pp. 269-316: 
que forman una isocolía, es decir, tienen el mismo o casi el mismo número de sílabas ${ }^{1}$. Así: sed et omne consilium consta de ocho sílabas, sed omnem dissipauerint tiene también ocho, sed omnem prorsus libidinem alcanza 9 sílabas. El último, reatu mortis adstringi, está limitado igualmente a 8 silabas. E1 período resulta, pues, estructurado a base de la combinación de miembros de ocho sílabas con miembros de 13 sílabas, como son: non solas manus in homicidio plecti de 13 , o quizás I2 śllabas, quod alterius animam inpegit in mortem de I4, o quizás I3 silabas, qui thura mensis adoleuere profanis de $\mathrm{I} 3$ sílabas. E1 penúltimo miembro extra uxorium torum et complexus licitos euagantem con sus I9 o quizás I7 silabas, no obsta a la armonía de proporciones en el conjunto; antes bien cumple la norma de que el último miembro, o a veces el penúltimo, sea mayor para así abrazar el resto del período ${ }^{2}$.

De estas consideraciones se desprende que el período no necesita mayor número de miembros y que la palabra original oculta bajo la corrupta no puede alargar el miembro sed omnem dissipauerint en más de una o a lo sumo dos sflabas, para no sobrepasar la longitud que le corresponde por la isocolía con los otros dos miembros introducidos por sed. La corrupción no está en dissipauerint, cuya sustitución no resuelve la falta de sentido. Por tanto omnem ha de ser enmendado por una palabra que no exceda de las cuatro sílabas.

\section{Consideraciones léxicas}

Las conjeturas propuestas hasta ahora parten de sed omnem y buscan en Tertuliano o Cipriano frases más o menos parecidas que empezando por sed omnem o sed omnes contengan algún pretérito perfecto en $u i$ de indicativo o mejor de subjuntivo. Un adjetivo como omnem parece demasiado corriente para no hacernos pensar en que se haya deslizado como lectio facilior en vez de la palabra que oculta. Además, su significado es muy pobre y genérico para que llamase la atención del

1 Sobre el concepto de isocolia, parisosis y paromóiosis puede verse Demetrio Falereo, Пєрl Épunvelós, pp. 22-25, ed. L. Radermacher, Stuttgart-Teubner, 1967, p. 9 s.; Cic., De Or. III, 54, 206; Ad Herenn. IV, 28; J. Martin, Antike Rhetorik, München, 1974, p. 310, y sobre todo, K. Polheim, Die lateinische Reimprosa, Berlin, 1925, p. 136 s. y 166. Puede verse también Th. Mommsen, "Zur lateinischen Stichometrie", Hermes 21,1885, p. 150, y en Gesammelte Schriften, Berlin, 1909, VII, p. 29r.

- Demetrio Falereo, op. cit., 18, p. 8; Cic., De Or. III 48, 186; K. Polheim, op. cit., pp. XI, I4, 34, I50, 185 s., 273. 
amanuense distrayéndole hasta el punto de hacerle copiar corruptamente un dissipauerint.

Con frecuencia el verbo dissipare tiene como complemento palabras que designan el cuerpo humano o sus miembros, ya sea $a$ ) en sentido físico, ya sea $b$ ) en sentido moral. En los escritores cristianos latinos antiguos es $\tan$ frecuente que E. Löfstedt ${ }^{1}$ llega a considerarlo como una especie de terminus technicus.

a) En sentido físico

๔) Escritores paganos:

dissipabo te tamquam folia farfari (Plaut., frg. inc. 50).

Marii sitas reliquias apud Anienem dissipari iussit Sulla uictor (Cic., Leg. II 56).

totos cum sanguine dissipat artus (Lucan. III 473).

\section{B) Escritores cristianos:}

Deus dissipauit ossa eorum (Vulg., Ps. 52, 6).

(uos)... caprorum reclamantium uiscera cruentatis oribus dissipatis (Arnob. V 19).

senatorum cadauera dissipari iussit (Lact., Inst. diu. VI 20).

\section{r) Tertuliano:}

Merece especial atención Tertuliano por la circunstancia de ser fuente de nuestro escritor ${ }^{2}$. Recogemos los siguientes ejemplos ${ }^{3}$ :

feris humana corpora dissiparentur (Spect. 12, 4).

in amphitheatro derosa et in suo sanguine squalentia corpora (Spect. 2I, 3).

Ipsis Bacchanalium furiis nec mortuis parcunt christianis, quin illos de requie sepulturae, de asylo quodam mortis, iam alios, iam nec totos auellant, dissipent, distrahant (Apol. 37, 2).

1 Tertullians Apologeticum textkritisch untersucht, Lund, 1915, pp. 43.45. En Kritische Bemerkungen zu Tertullians Apologeticum, Lund, 1918, p. 73 s., resume lo dicho en la investigación anterior.

2 L. Rubio Fernández, San Paciano. Obras, p. 31 s.

3 G. Cleason, Index Tertullianeus, Paris, 1974, I, p. 432. 
Hoc pro suis omni atrocitate dissipatis nemo umquam temptauit christianus (Apol. 46, 16).

Rutilius... tormentis dissipatus (Fag. 5, 3).

Namque illa (genera animalium) sicarium uariis et exquisitis et iam praeter naturam eruditis feris dissipant $(A n .33,5)$.

b) En sentido moral

\section{๔) Escritores paganos}

Entre las palabras que designan un cuerpo moral y aparecen como complemento de dissipare pueden citarse, entre otras, ciuitas, domus, equitatus, phalanx, respublica. Basten los ejemplos siguientes ${ }^{1}$ :

ciuitates dissipare (Firm. Mater., Math. VIII 27, 7).

dispersam et dissipatam esse rem publicam (Liv. II 28).

in medio secunda legio immissa dissipauit phalangem (Liv. XIV $4 \mathrm{I}$ ).

B) Escritores cristianos

De los complementos de dissipare que designan un cuerpo moral, llama especialmente la atención, por su frecuencia en los escritores latinos cristianos antiguos, el sustantivo ecclesiam. Así entre los muchos ejemplos que podrían citarse recuérdense éstos:

ecclesiam dissiparent (Lact., Inst. diu. IV 30, 4).

columbae non erant qui ecclesian, dissipauerunt (Aug., Ioh. 15, 12). ecclesiae corpus quod prius dissipatum et in diuersa laceratum in suam compaginem iuncturamque redigetur (Ruf. Apol., Adu. Hier. I 42).

De la expresión dissipare un cuerpo moral a ecclesiam dissipare no había más que un paso, si se tiene presente que la Iglesia era para los cristianos también un cuerpo moral o, como se ha llamado posteriormente, el cuerpo místico de Cristo.

1 ThLL V dissipo I A I b, cols. $14^{87}$ 8.; II B I, cul. 1492.

$\mathbf{3}$ 


\section{r) Cipriano}

Una carta de Cipriano ${ }^{1}$ tiene un interés particular por cuanto $\mathrm{Pa}$ ciano la conocía y en su correspondencia con Simproniano transcribió un pasaje de cierta extensión, como es éste: Nam quodam in loco ad Antonianum hoc modo scripsit (Cyprianus); "Additum est etiam Nouatiano tunc scribente et quod scripserat sua uoce recitante et Moyse tunc confessore, nunc iam martyre scribente ut lapsis infirmis et in exitu constitutis pax daretur. Quae litterae per totum mundum missae sunt et in notitiam ecclesiis omnibus perlatae sunt, ${ }^{2}$.

En esta misma carta Cipriano escribe un párrafo relativo, también como el nuestro de Paciano, a los pecadores que no merecen la pax, precisamente por estar separados de la Iglesia. Como en nuestro texto, se trata de idólatras: Apostatae en Cipriano: qui thura mensis adoleuere profanis en Paciano. Cipriano menciona a estos apostatae junto con los que son ecclesiam dissipantes. En Paciano sed omnem dissipauerint sigue a los qui thura mensis adoleuere profanis. E1 párrafo en cuestión de Cipriano reza así: Apostatae uero et desertores uel aduersarii et hostes et Christi ecclesiam dissipantes, nec si occisi pro nomine foris fuerint, admitti secundum Apostolum possunt ad ecclesiae pacem, quando nec spiritus nec ecclesiae tenuerint unitatem ${ }^{3}$. Se impone la conclusión de que $\mathrm{Pa}$ ciano conocía la expresión ecclesiam dissipare y a mayor abundamiento la había leído en un catálogo de pecados a continuación de los apostatae y enemigos de Cristo, es decir, según la concepción de la Iglesia primitiva, a continuación de los idólatras, los qui thura mensis adoleuere profanis.

\section{Consideraciones paleográficas}

La palabra omnem aparece con la abreviatura de $-m$ final omnē en los mss. R L V. Es tan rara la abreviación de omnis, por ejemplo, on̄s ${ }^{4}$ en $R$, que bien puede presumirse que no era costumbre del escriba. De ello se desprende que ōme o quizás om se hallaba en el modelo de R, según lo confirma, además, el require marginal de Florus, mencionado

1 Ep. 55, 5, 2.

2 Pac., Ep. III $5,4$.

Ep. 55, 29, 3 .

- Sólo en $E p$. III 4, r: $\mathrm{R}$ fol. $66 \mathrm{r}$, lín. 3, y en Ep. III 11, 2: R fol. $69 \mathrm{r}$, lin. 12 aparece $o \bar{m} s$. 
anteriormente ${ }^{1}$. Con la abreviatura de omnem, es decir, om̄e o om guarda un gran parecido la abreviatura de ecclesiam, o sea, ecc o ecea ${ }^{2}$. La ausencia de $l$ en la abreviatura que dio origen a la falta podría resultar confirmada quizás por el hecho de que en el ms. de la Reina de Suecia se lee una vez eccesia con la correspondiente enmienda floriana ecclesia ${ }^{3}$. De todos modos la abreviatura normal en $\mathrm{R}$ es eccta. En la lectura errónea influyó probablemente también el adjetivo omnem del miembro inmediato siguiente.

La corrupción que motiva transcribir la $e$ de ecc como una $o$ no es ajena al tipo de errores sufridos por el amanuense de $\mathrm{R}$ o de su modelo. Se lee $o$ por $e$ en $\mathrm{R}, a$ ) en posición inicial como opiscopatus por episcopatus ${ }^{4} ; b$ ) en interior, por ejemplo, laboros por labores ${ }^{5}$, apolliacos por apelliacos ${ }^{6}$, miscore por miscere ${ }^{7}$, habos por habes ${ }^{8}$, no por $n e^{9}$. Estos errores bastan para ilustrar la confusión de $o$ por $e$ en ecclesiam abreviado en $e c \bar{c}$ o $e c \bar{c} \bar{a}$. Una vez leída $o$ la $e$ inicial era muy fácil interpretar como $m$ las dos $c c$ con el signo abreviativo. El resto y el conjunto de la corrupción se explicaría también, en parte, por la preocupación inconsciente del copista de $\mathrm{R}$ o de su modelo por reunir letras en sílabas vagamente verosímiles formando palabras inteligibles a primera vista ${ }^{10}$.

\section{Interpretación}

E1 miembro sed omnem dissipauerint, restituido y fijado en sed ecclesiam dissipauerint se contrapone a qui thura mensis adoleuere profanis como una especie de pecado de idolatría del mismo modo como los miembros siguientes relativos al adulterio sed omnem prorsus libidinem.

1 Cf. en este artículo, p. 23.

2 L. Traube, Nomina Sacra, München, 1906 (omnis), pp. 246, 249, (ecclesia), pp. 245, 254; W. M. Lindsay, Notae Latinae, Cambridge, 1915 (omnis), p. 160, (ecclesia), pp. 396,424 .

s Ep. II 3, 3: $\mathrm{R}$ fol. 6I v, lín. 25.

- Ep. III r, 6: $\mathrm{R}$ fol. $64 \mathrm{v}$, lín. 3 .

5 Labores ex, laboros eras. o R, Ep. III 16, 2: fol. $7 \mathrm{I} r$, lín. 28.

- Ep. I 3, 2: R fol. $5^{8} \mathrm{v}$, lín. 12, puede verse también Angel Anglada, "La tradición manuscrita de Paciano de Barcelona», Emerita 35, 1967, p. 145 s.

7 Ep. III I, 6: R fol. $64 \mathrm{v}$, lin. 26.

8 Ep. III r 5, 5: $\mathrm{R}$ fol. $7 \mathrm{r} \mathrm{r}$, lín. 13.

- Ep. I 3, r: R fol. $5^{8}$ v, lin. 9.

10 Cf. J. Mallon, Pelégraphie romaine, C. S. I. C., Madrid, 1952, p. 149; Angel Anglada, "Le corruttele del membro Aquarum in penitis ignibus fuerit alla luce della metafora della febbre", Vetera Christianorum 14, 1977, p. 266. 
extra uxorium thorum et complexus licitos enagantem. Tertuliano nos ayuda a precisar el sujeto de sed ecclesiam dissipauerint. La especie más importante dentro de la idolatría y el pecado más grave en la antigüedad cristiana, junto con el homicidio y el adulterio, era la herejia, que a su vez era también una forma de adulterio. Paciano abunda en este sentido cuando escribe a Simproniano: adultera mulier haeretica congregatio est ${ }^{1}$. Son explícitos a este respecto los siguientes pasajes del De Praescriptione haereticorum: Hoc erunt haereses, non minus nouarum doctrinarum peruersitate ecclesiam lacessentes, quam tunc antichristus persecutionum atrocitate persequetur nisi quod persecutio et martyras facit. haeresis apostatas tantum ${ }^{2}$. En este pasaje Tertuliano usa lacessentes, sinónimo de dissipantes, como adjetivo de haereses. La herejía y la idolatria se igualan en este otro texto: neque... dubitare quis debet neque $a b$ idololatria distare haereses cum et auctoris et operis eiusdem sint cuius et idololatria... Itaque omne mendacium quod de Deo dicunt, quodammodo genus est idololatriae ${ }^{3}$. La herejía es considerada como adulterio en: Hos (los herejes) ut insigniores et frequentiores adulteros ueritatis nominamus ${ }^{4}$.

No constituye nuestro texto ninguna excepción al decir que los herejes despedazan a la Iglesia. San Agustín llama despedazadores de 1a Iglesia a los herejes cismáticos: Si ergo quando uult Deus concitare potestates aduersus haereticos, aduersus schismaticos, aduersus dissipatores ecclesiae, aduersus exsufflatores Christi, aduersus blasphematores baptismi, non mirentur, quia Deus concitat ut a Sara uerberetur Agar ${ }^{5}$. Anteriormente en el mismo comentario había escrito: Columbae non erant qui ecclesiam dissipauerunt ${ }^{6}$.

Así como Paciano relaciona con los idólatras a los herejes que dilaceran a la Iglesia, así Verecundo de Iunca une a los homicidas Caín y Lamech con los ecclesiam dissipantes. Dice: Secundum multiplicem intellectum his quoque concurrit, qui Christi Ecclesiam dissipantes multos secum scissa caritate seducunt. Quos doctorum delicto grauari iste uersiculus terribiliter monstrat. Unde quaesumus uos, uarietatum doctrinis aliquando subuersos, ne putetis impune simplicitate deludi, aestimantes nihil uobis concurrere detrimenti, per alios oberrantes non propia uoluntate

Ep. III 22, 2.

4. 5 (CCh I, p. 190).

3 40, 8-10 (CCh I, p. 22I).

- 30, 1o (CCh I, p. 2 I I); véase, además, Tert., Idolol. I I-2; 7, 3; II, 5 (CCh II, pp. IIOI, IIO6, IIII); también, F. Cavallera, "La doctrine de la pénitence au III sièclen, $B L E 31,1930$, p. 57 .

- Ioh. I1, 13 (CCh XXXVI, p. I18).

- Ibid., 5, 12 (CCh XXXVI, p. 47). 
labentes: quasi inter Cain et Lamech homicidas sit ulla distantia, cum inuentor mali adsectatorque pari poena multetur et Dathan atque Abiron deceptique ab eis uno interitu deglutiti ${ }^{1}$.

Este gran celo por mantener la unidad de la Iglesia y huir de toda división remonta a los tiempos más primitivos del cristianismo. Ya la Didaché insistía en la unidad de la Iglesia y pedía al Señor que la aunara desde los cuatro vientos ${ }^{2}$. Recomendaba igualmente al fiel cristiano que no se apasionara en seguir doctrinas ${ }^{3}$. La recensión $\mathrm{Ca}$ mino de la Vida pone en boca de Kefas el precepto de no hacer escisión:

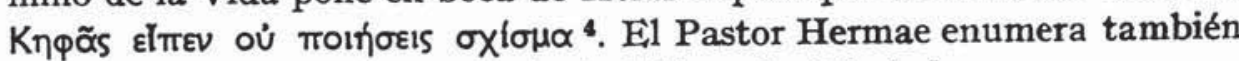
entre los pecados más graves la traición a la Iglesia ${ }^{5}$.

El texto que nos ocupa es un catálogo de pecados, que de una parte conserva la doble división en pecados de obra non solas manus in homicidio plecti y pecados de intención sed et omne consilium. quod alterius animam inpegit in mortem. Sigue también la tradición de otra distinción doble entre pecados ad mortem, reatu mortis adstringi y pecados que no son ad mortem. La enumeración de homicidio, idolatría y herejía y adulterio, estos dos últimos como especies menos patentes de adoración a los dioses paganos, dejan traslucir la forma antigua del catálogo triple. Todas estas circunstancias sitúan al escritor barcelonés dentro de una tradición literaria que en la predicación sobre la penitencia introduce un catálogo de pecados. El contenido de éste en nuestro pasaje sigue los puntos y aspectos formulados por la antigüedad cristiana y los interpreta con las expresiones propias de la literatura cristiana anterior y comunes en la oratoria penitencial ${ }^{6}$.

\section{Conclusión}

Las consideraciones precedentes garantizan con toda certeza la enmienda ecclesiam corrigiendo omnem que mantiene la línea tradicio-

1 Cant. Hier. 5, 7 (CCh XCIII, p. 72).

29,$4 ; 10,5$.

3,3 .

- ro.

5 $8,6,4 ; 9$, I9, I; cf., también, A. Vanbeck, "La Pénitence dans le Pastear d'Hermas", RHLR 2, I9II, pp. 395-397.

- Sobre los catálogos de pecados puede verse el articulo Pénitence de $\mathbf{E}$. Amman, en DThC XII I y A. Vögtle, Achtlasterlehre, LAC I, cols. 74-79. Puede verse otra interpretación de nuestro pasaje a base del texto de Peyrot en E. Göller, Analekten zur Bussgeschichte des vierten Jahrhundertss, $R Q$ 26, 1928, pp. 253 s. 
ŚNGEL ANGLADA

nal del léxico en el contexto de dissipare y dentro del contexto del doble y triple catálogo de pecados en la literatura latina cristiana antigua. Por lo que se refiere a la compositio, el número de ślabas que alcanza el miembro corregido guarda la isocolia con los otros miembros paralelos. La reconstrucción paleográfica del error explica a plena satisfacción cómo el copista llegó a equivocarse. En consecuencia, el texto queda fijado con toda seguridad en estos términos: sed ecclesiam dissipauerint.

ANGel ANglada

Barcelona 